\title{
Avaliação Psicológica de Crianças com Suspeita de TEA: Perfil Interativo dos Avaliadores
}

\author{
Gabriela Moreira Romeira1 (D), Letícia Backes Schreiner (D), Cleonice Alves Bosa (D) \\ Universidade Federal do Rio Grande do Sul, Porto Alegre-RS, Brasil
}

\section{RESUMO}

O objetivo deste estudo é o de investigar o perfil interativo dos avaliadores durante a hora lúdica diagnóstica na avaliação de crianças com suspeita de TEA. Participaram nove avaliadoras em interação individual com 22 crianças (de 24 a 74 meses), durante uma sessão de administração do Protocolo de Avaliação Comportamental para Crianças com Suspeita de TEA - Versão Revisada - Não Verbal (PROTEA-R-NV), que foi videogravada. Utilizou-se o Manual de Codificação dos Estilos Interativos do Avaliador, construído para este estudo. Dois juízes independentes e cegos aos objetivos do estudo registraram a ocorrência, a não ocorrência ou a não aplicação de três categorias de responsividade do avaliador: responsivo, diretivo e intrusivo. Os resultados demonstraram que as avaliadoras tenderam a adotar um estilo interativo diretivo, seguido pelos estilos responsivo e intrusivo. Discute-se que as características da criança e o treinamento prévio das avaliadoras na administração do instrumento pode ter influenciado estes resultados.

Palavras-chave: transtorno autístico; avaliação psicológica; método qualitativo; desenvolvimento.

\section{ABSTRACT - Psychological assessment of children with suspected ASD: Interactive profile of the evaluators}

This study aimed to investigate the evaluators' interactive style profiles during the diagnostic assessment of children with suspected ASD. Two independent judges coded videos of assessment sessions of 22 children (aged from 24 to 74 months), using the Behavioral Evaluation Protocol for Children at Risk for ASD - Revised Version - Non-Verbal (PROTEA-R-NV), interacting with nine different evaluators. The independent judges used the Evaluators' Interactive Styles Coding Manual to encode the behaviors observed. The unit of analysis was a one-minute interactive episode of each videotape. Each judge registered the presence or absence of the evaluator's behavior described in the manual, when applicable. The evaluators had a strong tendency to adopt directiveness as an interactive style, followed by responsiveness and intrusiveness. It was concluded that the child's characteristics and the evaluator's previous training in the administration of the instrument may have influenced these results.

Keywords: autistic disorder; psychological assessment; qualitative method; development.

\section{RESUMEN - La evaluación psicológica de niños con sospecha de TEA: Perfil interactivo de los evaluadores}

El objetivo de este estudio es investigar el perfil interactivo de los evaluadores durante el proceso de evaluación en la hora lúdica diagnóstica de niños con sospecha de TEA. Nueve evaluadores participaron en la interacción individual con 22 niños (de 24 a 74 meses) durante la administración del Protocolo de Evaluación Conductual para Niños con Sospecha de TEA - Versión Revisada No verbal (PROTEA-R-NV), que fue grabada en video. Se utilizó el Manual de Codificación de Estilos Interactivos del Evaluador, creado para este estudio. Dos jueces independientes desconocedores de los objetivos del estudio registraron la ocurrencia, no ocurrencia o no aplicación de tres categorías de capacidad de responsabilidad del evaluador: receptivo, directivo e intrusivo. Los resultados confirmaron que los evaluadores tendían a adoptar un estilo interactivo Directivo, seguido de los estilos Receptivo e Intrusivo. Se concluye que las características de los niños y la capacitación previa de los evaluadores en la administración del instrumento pueden haber influido en estos resultados.

Palabras clave: trastorno del espectro autista; evaluación psicológica; método cualitativo; desarrollo.

O Transtorno do Espectro Autista (TEA) é uma condição neurodesenvolvimental que tem, entre suas características, baixa reciprocidade na interação social, estereotipias motoras, exploração incomum de objetos e adesão inflexível a rotinas (American Psychiatric Association [APA], 2013; Teixeira et al., 2017). Além disso, algumas crianças com TEA podem apresentar hipo ou hipersensibilidade a estímulos simultâneos, o que pode resultar em uma sobrecarga sensorial (APA, 2013). Os comportamentos podem variar, tanto na frequência quanto na gravidade, sendo os primeiros anos de vida o período crítico para o início dos sinais de risco (Mugzach et al., 2015).

Uma das dificuldades mais comuns, no que se refere aos comportamentos sociocomunicativos em TEA, é a de Atenção Compartilhada (AC), sendo o comprometimento dessa habilidade um dos principais sinais de risco para o transtorno, uma vez que, conforme as teorias interacionistas, a AC é a base para o desenvolvimento social e linguístico subsequente (Tomasello, 1999/2003). Crianças com TEA tendem a apresentar

${ }^{1}$ Endereço para correspondência: Rua Gonçalo de Carvalho, 199, apto 302, 90035-170, Porto Alegre, RS. Tel.: (51) 99215-4876. E-mail: gabriela.romeira@gmail.com Artigo derivado da Dissertação de mestrado de Gabriela Moreira Romeira com orientação de Cleonice Alves Bosa, defendida em 2019 no Programa de PósGraduação em Psicologia da Universidade Federal do Rio Grande do Sul. 
compartilhamento reduzido de interesses e emoções com outras pessoas (APA, 2013), buscando o adulto principalmente para obter assistência, nem sempre com a intenção de interagir socialmente. Outro déficit bastante frequente em indivíduos com TEA refere-se à habilidade de simbolizar. Conforme Tomasello (1999/2003), compreender a si e aos outros como agentes intencionais permite que as crianças participem de cenas de AC, que são fundamentais à aquisição dos símbolos linguísticos e encontram-se atreladas às bases da interação social. Em conjunto com a AC, déficits no jogo simbólico têm sido considerados um dos indicadores precoces mais robustos de TEA (Zyga et al., 2015).

Do ponto de vista neurodesenvolvimental, a literatura recente tem demonstrado também que crianças com TEA podem apresentar alterações em funções executivas (FE), o que também auxilia na compreensão da rigidez comportamental durante a interação social e a brincadeira (Seabra et al., 2017). Um estudo brasileiro comparou o desempenho das FE de crianças e adolescentes com TEA ao de um grupo controle com desenvolvimento típico (Czermainski et al., 2014). Os autores concluíram que o grupo com TEA apresentou desempenho inferior em todas as tarefas que avaliaram as FE (e.g., planejamento, flexibilidade cognitiva, controle inibitório, fluência verbal semântica e fonológica e memória de trabalho verbal e visuoespacial). Esses resultados demonstram que as dificuldades comportamentais das crianças com TEA podem influenciar a qualidade da interação social com os seus cuidadores, especialmente a sua responsividade durante a brincadeira.

O núcleo da responsividade, por sua vez, é a sincronia. De acordo com teorias que estudam as interações mãe-criança, a sincronia é definida pela adaptação comportamental dinâmica e recíproca entre os parceiros envolvidos na interação (Leclère et al., 2014). Esse fenômeno inclui comportamentos comunicativos verbais $\mathrm{e}$ não verbais, além de comportamentos emocionais (e.g., gestos, olhares, expressões faciais), bem como implica interações necessariamente coordenadas entre os parceiros (Delaherche et al., 2012). A qualidade da responsividade do adulto pode ser identificada, na literatura, por meio de diferentes estilos interativos. Há várias décadas, o estilo interativo diretivo tem sido identificado na literatura como favorecedor da interação. Por exemplo, um estudo de Aquino e Salomão (2005) analisou possíveis variações de comportamentos diretivos maternos em 16 díades mãe-criança, com idades entre 24 e 30 meses. Os resultados revelaram que comportamentos maternos que dirigiam a atenção infantil foram utilizados em momentos nos quais a criança não estava atenta ao objeto a que a mãe se referia, resultando, consequentemente, no engajamento infantil.

O compartilhamento de tópico é outro estilo interativo da responsividade, envolvendo comportamentos que buscam seguir o mesmo foco de atenção da criança.
Há uma continuidade de ações e comportamentos já iniciados de forma espontânea pela criança, em atividades ou brincadeiras mediadas por objetos (Bosa \& Souza, 2007). Comportamentos contingentes de compartilhar tópicos de interesse têm sido apontados há várias décadas como preditores do processo subsequente da aquisição da linguagem no desenvolvimento típico (Akhtar \& Tomasello, 1996). Um estudo de Meimes (2014) investigou a relação dos estilos de interação (diretivo, intrusivo e de compartilhamento de tópico) de mães de meninos com TEA e sua influência nos comportamentos infantis (engajamento e não engajamento). Participaram do estudo quatro díades mãe-criança com TEA, sendo que, em duas díades, o estilo de compartilhamento de tópico materno resultou em comportamentos de engajamento infantil; contudo, nas outras duas, não houve engajamento por parte da criança. De forma contrária aos estilos diretivo e de compartilhamento de tópico, a literatura demonstra que o estilo intrusivo do adulto tende a resultar em comportamentos de protesto e afastamento da interação por parte da criança (Bosa \& Souza, 2007). A intrusividade consiste na presença de comportamentos repressores do adulto em relação à criança, podendo inibir, assim, a sua espontaneidade durante a interação (Bosa \& Souza, 2007). Comportamentos intrusivos são comumente confundidos com diretividade, uma vez que também dirigem ou controlam as ações da criança (Bosa, 1998). No entanto, a principal diferença entre esses dois estilos interativos está na presença (i.e., diretividade) ou na ausência (i.e., intrusividade) de sensibilidade do adulto em relação aos desejos e necessidades da criança (Meimes, 2014), denotando pouca responsividade. Dessa forma, deve-se sempre considerar o propósito do comportamento para caracterizá-lo como intrusivo ou diretivo, ressaltando-se que o primeiro (intrusivo) tende a dificultar o engajamento da criança em situações interacionais. Especificamente no TEA, com relação ao manejo, a intrusividade pode manifestar-se, também, em comportamentos nos quais o parceiro social da criança a estimula para além do que ela é capaz de suportar (i.e., superestimulação). É preciso considerar as dificuldades dessas crianças frente a estímulos sensoriais simultâneos e/ou demanda social excessiva (APA, 2013).

Destaca-se que, na literatura em desenvolvimento típico, o estudo de Paavola- Ruotsalainen et al. (2017) demonstrou que comportamentos responsivos por parte do adulto foram positivamente relacionados às habilidades de compreensão do contexto por parte da criança. A responsividade do adulto também influenciou positivamente as habilidades de cunho simbólico e gestos comunicativos, apontando para a importância de estudos sobre a influência da qualidade do estilo interativo do adulto para o desenvolvimento infantil.

Embora a literatura seja farta na investigação da interação social pais-criança, existem outros contextos em que a observação da interação entre um adulto e a criança 
é fundamental. Um exemplo é o uso da observação clínica em contextos de caráter lúdico (Marques \& Bosa, 2015; Volkmar et al., 2004), comumente conhecida como hora do jogo, hora lúdica ou entrevista lúdica diagnóstica (Krug \& Bandeira, 2016), na avaliação psicológica.

Bosa e Zanon (2016) destacam que os sinais mais robustos podem ser reconhecidos durante a atividade lúdica. Trata-se de comportamentos que se constituem em risco para o transtorno, como prejuízos na comunicação e na interação social, além da ausência de habilidades simbólicas (Zanon et al., 2014). Ainda, reitera-se que o propósito da interação no contexto avaliativo não concerne apenas à identificação dos comprometimentos desenvolvimentais, mas também das potencialidades infantis. Logo, é fundamental que o profissional esteja atento às necessidades da criança, adequando o seu manejo para que essas potencialidades possam ser manifestadas (Bosa et al., 2017).

Nesse sentido, considera-se que a brincadeira constitui uma ferramenta fundamental a ser utilizada pelo avaliador para mediar sua interação com a criança, proporcionando, assim, um contexto favorável à emergência dos comportamentos a serem analisados (Bosa et al., 2017). Ressalta-se, no entanto, que os instrumentos comumente utilizados na avaliação de crianças com TEA, principalmente os que estruturam a hora lúdica, embora orientem os comportamentos do adulto, dão pouca ênfase aos estilos interativos do avaliador. Bosa et al. (2017) atentam para o fato de que os estilos interativos do avaliador podem influenciar a frequência e a qualidade das respostas infantis, sendo o conhecimento acerca desses estilos particularmente importante para que os profissionais que atuam na área possam engajar socialmente crianças com TEA durante a avaliação.

No entanto, embora haja evidências empíricas sobre os comportamentos que promovem a sustentação da interação cuidador-criança com TEA (Dos Santos et al., 2015), pouco se sabe sobre as características dos comportamentos do avaliador, em situação de hora lúdica diagnóstica, para além das publicações com enfoque psicodinâmico. É fundamental, portanto, conhecer os estilos interativos que tendem a predominar durante a hora lúdica pelo potencial que alguns comportamentos apresentam na promoção do engajamento infantil (Bosa et al., 2017).

A revisão da literatura demonstra a carência de estudos que investiguem o comportamento do avaliador em contexto psicodiagnóstico, na área do TEA, sobretudo aqueles voltados à identificação precoce. O não engajamento da criança em interações sociais durante a avaliação compromete a observação clínica do profissional, bem como os resultados do processo avaliativo.

Dessa forma, o objetivo do estudo foi investigar o perfil interativo dos avaliadores durante a hora lúdica na avaliação de crianças com suspeita de TEA. A expectativa foi a de identificar estilos interativos predominantemente responsivos e diretivos e com menor ocorrência do estilo intrusivo. O projeto em que este estudo se insere articula pesquisa e extensão e ocorre em uma clínica-escola. Os avaliadores receberam treinamento teórico-prático, previamente à avaliação de crianças com suspeita de TEA. As intervenções do avaliador ocorreram sob a supervisão de um professor orientador.

Do ponto de vista das implicações da pesquisa para a prática clínica, pretende-se contribuir para o conhecimento dos comportamentos do adulto que a literatura aponta como favorecedores da interação social de crianças com TEA. Esses comportamentos dizem respeito a perfis interativos mais responsivos e diretivos e menos intrusivos. Com isso, pretende-se reduzir as situações interativas assincrônicas na brincadeira (protesto, afastamento etc.), tanto em contexto lúdico de avaliação psicológica, quanto de intervenção, e auxiliar na promoção da emergência das potencialidades infantis. Esse conhecimento pode, ainda, fundamentar programas de formação profissional em avaliação psicológica na área do TEA e aqueles direcionados ao treinamento de pais.

\section{Método}

\section{Delineamento e Participantes}

Estudo empírico retrospectivo, descritivo e transversal, por meio de um banco de dados composto por 22 videogravações de sessões de avaliação da interação avaliador-criança com suspeita de TEA. As crianças foram avaliadas em duas sessões, mas somente uma, de cada criança, foi utilizada. O critério de opção por apenas uma sessão foi o de conveniência, adequando-se o tempo envolvido na codificação ao período destinado à coleta de dados (seis meses). Optou-se, então, pela segunda sessão a fim de controlar a influência da maior dificuldade de adaptação da criança a um ambiente não familiar. As sessões ocorreram em contexto de hora lúdica diagnóstica, em uma clínica-escola. Participaram nove avaliadoras, equiparadas quanto à faixa de idade $(m=26,44$; $d p=4,27$ ), escolaridade (quatro com ensino superior em andamento; cinco com ensino superior concluído) e duração/qualidade do treinamento (supervisionado). As avaliadoras fazem parte da clínica-escola, e o treinamento para a avaliação das crianças (20hrs) envolveu seminários teóricos sobre transtorno do espectro autista, teorias interacionistas sociopragmáticas, observação da interação criança-avaliador e prática de interação criança-avaliador em situação de hora lúdica. A qualidade do treinamento foi observada por meio da avaliação da fidedignidade entre avaliadoras na aferição do instrumento (índice de $90 \%$ de concordância em relação à avaliadora sênior) e da supervisão individual (no caso da administração) por um professor especialista. As 22 crianças (três do sexo feminino e 19 do sexo masculino), avaliadas nas sessões, tinham idades entre 24 e 74 meses ( $m=45,95 ; d p=15,37)$. Todas apresentaram comportamentos que preenchem 
critérios para o diagnóstico de TEA, conforme o DSM-5 e o PROTEA-R-NV, além de dificuldades na linguagem expressiva e compreensiva, de acordo com os registros clínicos. Foram incluídas as videogravações das crianças, cujos pais assinaram o Termo de Consentimento Livre e Esclarecido (TCLE) e que possuíam prontuário completo, contendo as informações necessárias para o estudo (e.g., dados sociodemográficos e clínicos). A autorização de acesso aos vídeos, pelos responsáveis, deu-se via consentimento livre e esclarecido específico para as imagens. Foram excluídas: (a) as videogravações que não corresponderam aos critérios de qualidade da filmagem (tempo na íntegra, resolução e qualidade sonora); (b) sessões em que as avaliadoras não preenchiam os critérios estabelecidos para este estudo (e.g., equiparação quanto ao sexo, faixa etária e escolaridade) e (c) comorbidades registradas no prontuário (deficiência intelectual grave, comprometimentos sensoriais não corrigidos ou outros transtornos do neurodesenvolvimento).

\section{Instrumentos e Materiais}

Termo de Consentimento Livre e Esclarecido (TCLE). Autorização do uso dos dados gerados pela pesquisa na composição do banco de dados do grupo de pesquisa e sua utilização em estudos futuros.

Ficha sobre dados sociodemográficos da família e informações clínicas sobre a criança com TEA. Informações sociodemográficas da família, desenvolvimento da criança e aspectos clínicos relacionados ao TEA, com base nos registros a partir da entrevista de anamnese da criança.

Ficha sobre dados sociodemográficos e informações sobre o avaliador. Informações sobre idade, sexo, escolaridade e informações sobre a formação do avaliador (e.g., tipo e tempo de experiência na avaliação de crianças com suspeita de TEA).

Protocolo de Avaliação Comportamental para Crianças com Suspeita de TEA - Versão Revisada - Não Verbal (PROTEA-R-NV) (Bosa, 1998; Bosa \& Salles, 2018; Bosa et al., 2016; Marques \& Bosa, 2015). Integra o Sistema PROTEA-R de Avaliação do Transtorno do Espectro Autista, que é um instrumento que sistematiza as entrevistas e a observação clínica de casos com suspeita de TEA e de outros transtornos da comunicação na infância. É composto por 17 itens e avalia a qualidade e a frequência de comportamentos característicos do TEA (i.e., comprometimentos sociocomunicativos, qualidade da brincadeira e presença de comportamentos repetitivos e estereotipados). É administrado e codificado a partir da observação clínica da criança em situação de brincadeira com o avaliador em dois contextos: um livre e outro semiestruturado, com duração média de 45 minutos.

Manual de Codificação dos Estilos Interativos dos Avaliadores. Elaborado especialmente para o presente estudo, com base na literatura e na experiência clínica dos pesquisadores. O manual é composto por: (a) definições operacionais de cada estilo interativo (categorias de responsividade, diretividade e intrusividade); (b) os comportamentos ilustrativos de cada um destes (subcategorias); e (c) por uma Folha de Registro dos Comportamentos dos Avaliadores, que contém as 17 subcategorias de comportamento do contexto de brincadeira livre e 14 do contexto de brincadeira semiestruturada, para cada um dos 17 episódios de um minuto e dos 22 casos. Foi preenchida por cada uma das juízas, contendo um total de 5896 comportamentos codificados quanto à sua presença ou ausência.

\section{Procedimentos e Considerações Éticas}

O banco de dados advém de uma pesquisa anterior do grupo de pesquisa, aprovada pelo Comitê de Ética em Pesquisa (CEP) do Instituto de Psicologia da Universidade Federal do Rio Grande do Sul (UFRGS) sob o protocolo $n^{\circ}$ 06632012.4.0000.5334. A observação do contexto de brincadeira livre iniciou no momento em que o avaliador dirige a atenção da criança para um dos brinquedos dispostos no chão ou na caixa transparente e terminou 15 minutos após seu início. No contexto de brincadeira semiestruturada, foi analisado o primeiro minuto, após a apresentação de cada brinquedo, por considerar esse um tempo suficiente para analisar os comportamentos do avaliador na apresentação de cada objeto. As duas juízas receberam treinamento (4 horas): (1) teórico, em que foram discutidas noções sobre TEA, teoria sociopragmática de Tomasello e demais pressupostos teóricos que embasaram a construção do Sistema PROTEA-R de Avaliação do TEA, sendo apresentado o Manual de Codificação dos Estilos Interativos dos Avaliadores e o Protocolo de Codificação dos Comportamentos dos Avaliadores; e (2) prático, no qual foi realizada a codificação dos comportamentos de uma díade piloto, utilizando-se o Manual e o Protocolo.

\section{Análise de Dados}

Os vídeos foram analisados utilizando-se os procedimentos de observação sistemática (Bakeman \& Gottman, 1986). Os dados foram examinados a partir de estatística descritiva, utilizando-se o software SPSS Statistics Editor (IBM, 2016).

\section{Resultados}

Para cada uma das 22 videogravações, nos episódios de interação do contexto de brincadeira livre, observou-se a presença, a ausência ou não aplicação (quando não foi possível codificar por problemas técnicos etc.) de três categorias de responsividade do avaliador: Responsividade, Diretividade e Intrusividade. A categoria Responsividade incluía comportamentos, como seguir o mesmo foco de atenção da criança ou antecipar uma ação que seria realizada pelo avaliador a seguir; a categoria Diretividade envolvia comportamentos 
de dirigir a atenção da criança a alguma atividade ou demonstrar operacionalmente como se utilizava um brinquedo, por exemplo. E a categoria Intrusividade diz respeito aos comportamentos que não consideraram as aparentes necessidades e desejos da criança.

Já nos episódios de interação do contexto de brincadeira semiestruturada, observou-se a presença ou ausência de: seis comportamentos referentes ao estilo interativo de Responsividade; três comportamentos do estilo interativo Diretividade; e cinco comportamentos de Intrusividade (Tabela 1).

A fidedignidade entre as juízas foi analisada por meio do cálculo do índice de concordância entre juízes com base na seguinte razão: concordâncias/(concordâncias + discordâncias) X 100 (Fagundes, 2015), devendo estes atingirem um índice de 70\%, ou superior. Encontrou-se um índice de concordância (IC) de 78,66\%, considerando o total de comportamentos codificados (268) em cada uma das 22 videogravações. Com relação ao contexto de brincadeira livre, especificamente, o IC foi de $77,46 \%$. No contexto de brincadeira semiestruturada, as juízas atingiram $80,60 \%$ de concordância. Para a identificação dos perfis das avaliadoras, foram consideradas as concordâncias entre as juízas $(78,66 \%$ do total de comportamentos codificados). Quando houve discordâncias, uma terceira juíza, especialista na área, dirimiu-as.

Em cada uma das 22 videogravações, observou-se a presença, a ausência ou falta de aplicação de 268 comportamentos, totalizando 5896 comportamentos observados, que foram codificados pelas duas juízas. $\mathrm{O}$ índice de concordância total para todos os casos ficou dentro da faixa estipulada como aceitável neste estudo (de 70 a $100 \%)$.

Com relação aos perfis de estilos interativos observados nas 22 videogravações, foram calculadas as médias e desvios padrão das frequências da presença de cada comportamento, apenas. Os resultados revelaram que o estilo interativo que mais predominou em ambos os contextos de brincadeira foi a Diretividade, seguida pela Responsividade e pela Intrusividade. Foram calculadas as médias das frequências de cada estilo interativo a partir da razão entre a frequência total e o número de itens (comportamentos) de cada estilo. As médias variam de 0 a 1 e foram as seguintes: Diretividade $(m=0,48)$; Responsividade $(m=0,28)$; Intrusividade $(m=0,02)$. A Tabela 1 apresenta os valores das médias e desvios padrão de cada um dos estilos interativos, em cada um dos contextos de brincadeira.

Tabela 1

Médias e Desvios Padrão da Frequência dos Estilos Interativos nos Contextos de Brincadeira Liure e Semiestruturada

\begin{tabular}{|c|c|c|c|c|c|c|}
\hline \multirow{2}{*}{ Estilo interativo } & \multicolumn{3}{|c|}{ Contexto de brincadeira livre } & \multicolumn{3}{|c|}{ Contexto de brincadeira semiestruturada } \\
\hline & Mínimo & Máximo & Média (DP) & Mínimo & Máximo & Média (DP) \\
\hline Responsividade & 0,20 & 0,39 & $0,28(0,04)$ & 0,07 & 0,33 & $0,20(0,07)$ \\
\hline Diretividade & 0,05 & 0,70 & $0,48(0,17)$ & 0,71 & 1,00 & $0,93(0,08)$ \\
\hline Intrusividade & 0,00 & 0,07 & $0,02(0,23)$ & 0,00 & 0,17 & $0,03(0,04)$ \\
\hline
\end{tabular}

O teste de Wilcoxon revelou que, no contexto de brincadeira livre, a média de Diretividade foi significativamente mais alta que a média de Responsividade $(Z=-3,49, p<0,001)$; e Responsividade significativamente mais alta que Intrusividade, $(Z=-4,11, p<0,001)$. Já no contexto de brincadeira estruturada, a média da Diretividade foi também significativamente mais alta que a média de Responsividade $(Z=-4,11, p<0,001)$. A média de Responsividade significativamente mais alta que a de Intrusividade $(Z=-4,06, p<0,001)$.

Por outro lado, quando consideradas todas as codificações (SIM, NÃO e NÃO SE APLICA), nos casos em que houve concordância entre as juízas (78,66\%), foram calculadas as frequências da presença de cada um dos comportamentos codificáveis. No contexto de brincadeira livre, cada um dos comportamentos poderia estar presente em cada um dos dez episódios interativos de cada um dos 22 casos. Para tanto, cada comportamento tinha a possibilidade de estar presente 220 vezes. Os resultados revelaram que, nesse contexto de brincadeira, em relação ao estilo responsivo, os comportamentos de observar o contexto $(90,9 \%)$ e compartilhar tópico $(73,63 \%)$ foram os mais frequentes. No estilo Diretivo, os comportamentos que mais ocorreram foram os de tornar as brincadeiras atrativas $(75,45 \%)$ e os de dirigir a atenção $(74,54 \%)$. No estilo Intrusivo, embora todos os comportamentos tenham ocorrido com frequência muito baixa, o comportamento mais presente foi o de desconsiderar interesses $(4,09 \%)$ e os de fazer perguntas em excesso $(3,18 \%)$.

Já no contexto de brincadeira semiestruturada, cada comportamento poderia estar presente em cada um dos sete episódios interativos de cada um dos 22 participantes. Assim, cada comportamento tinha a possibilidade de estar presente 154 vezes. O comportamento mais frequente do estilo Responsivo foi compartilhar tópico $(57,79 \%)$. No estilo Diretivo, todos os comportamentos ocorreram com frequência similar, destacando-se o de dirigir a atenção $(98,70 \%)$; já no estilo Intrusivo, todos os comportamentos apresentaram baixas frequências, sendo não antecipar ações a que mais ocorreu (9,09\%). 
A Tabela 2 apresenta as frequências porcentagens de cada comportamento em ambos os contextos de brincadeira.

Foram também exploradas eventuais diferenças nos comportamentos dos estilos interativos entre os dois contextos (brincadeira livre: BL e semiestruturada: BSE). A Tabela 2 demonstra que, na comparação de cada um dos comportamentos que constituem cada categoria de estilo interativo (Responsividade, Diretividade e Intrusividade), nos dois contextos, observa-se o seguinte: (a) houve tendência de maior diferença na ocorrência de um dos comportamentos responsivos (Antecipar ações - AA; BL: $f=9$; BSE: $f=52$ ) no contexto semiestruturado do que no de brincadeira livre; (b) houve tendência de maior diferença na ocorrência de comportamentos diretivos no contexto semiestruturado do que no de brincadeira livre. $\mathrm{O}$ avaliador tendeu a dirigir mais atenção (DA; BL: $f=164$; BSE: $f=152$ ), demonstrar como se opera um objeto (DOO; BL: $f=87$; BSE: $f=131$ ) e tornar as brincadeiras mais atrativas (TBA: BL: $f=166$; BSE: $f=149$ ); (c) houve tendência de maior diferença na ocorrência de um comportamento intrusivo (Realizar ações sem antecipação - ASA; BL: $f=6$; BSE: $f=14$ ) no contexto semiestruturado do que no de brincadeira livre. Por outro lado, no contexto de brincadeira livre, os seguintes comportamentos tenderam a ocorrer mais do que no semiestruturado: Responsividade Compartilhar tópico (CT; BL: $f=162$; BSE: $f=89$ ) e respeitar o tempo de resposta (RTR; BL: $f=17$; BSE: $f=7$ ); Intrusividade: fazer perguntas em excesso (FPE; BL: $f=7$; BSE: $f=1)$.

Tabela 2

Frequências e Porcentagens dos Comportamentos Codificáveis em Ambos os Contextos de Brincadeira (Liure, N=900; Semiestruturada $\mathrm{N}=648$ )

\begin{tabular}{|c|c|c|c|c|c|c|}
\hline & \multicolumn{3}{|c|}{ Contexto de brincadeira livre } & \multicolumn{3}{|c|}{$\begin{array}{c}\text { Contexto de brincadeira } \\
\text { semiestruturada* }\end{array}$} \\
\hline & Comportamento & $f(\%)$ & $n$ & Comportamento & $f(\%)$ & $n$ \\
\hline \multirow{8}{*}{ 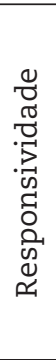 } & Observar o contexto (OC) & $200(90,90)$ & & $(\mathrm{CT})$ & $89(57,79)$ & \\
\hline & Compartilhar tópico (CT) & $162(73,63)$ & & $(\mathrm{AA})$ & $52(33,76)$ & \\
\hline & Aproximar-se gradualmente (AG) & $37(16,81)$ & & $(\mathrm{AG})$ & $26(16,88)$ & \\
\hline & Respeitar o tempo de resposta (RTR) & $17(7,72)$ & & $(\mathrm{AP})$ & $9(5,84)$ & \\
\hline & Atentar a pedidos de assistência (AP) & $11(5,00)$ & & (RTR) & $7(4,54)$ & \\
\hline & Antecipar ações (AA) & $9(4,09)$ & & $(\mathrm{RP})$ & $7(4,54)$ & \\
\hline & Respeitar pausas interacionais (RP) & $8(3,63)$ & & & & \\
\hline & & & 444 & & & 190 \\
\hline \multirow{5}{*}{ 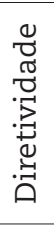 } & Tornar as brincadeiras atrativas (TBA) & $166(75,45)$ & & (DA) & $152(98,70)$ & \\
\hline & Dirigir a atenção (DA) & $164(74,54)$ & & $(\mathrm{TBA})$ & $149(96,75)$ & \\
\hline & Demonstrar como se opera um objeto (DOO) & $87(39,54)$ & & (DOO) & $131(85,06)$ & \\
\hline & Engajar socialmente (ES) & $11(5,00)$ & & & & \\
\hline & & & 428 & & & 432 \\
\hline \multirow{7}{*}{ 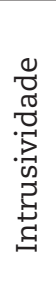 } & Desconsiderar interesses (DCI) & $9(4,09)$ & & (ASA) & $14(9,09)$ & \\
\hline & Fazer perguntas em excesso (FPE) & $7(3,18)$ & & $(\mathrm{DCI})$ & $8(5,19)$ & \\
\hline & Realizar ações sem antecipação (ASA) & $6(2,72)$ & & $(\mathrm{DRP})$ & $3(1,94)$ & \\
\hline & Aproximar-se abruptamente (AAb) & $2(0,90)$ & & $(\mathrm{FPE})$ & $1(0,64)$ & \\
\hline & Desrespeitar o tempo de resposta (DTR) & $2(0,90)$ & & (DTR) & $0(0,00)$ & \\
\hline & Desrespeitar pausas interacionais (DRP) & $2(0,90)$ & & & & \\
\hline & & & 28 & & & 26 \\
\hline
\end{tabular}

Nota. ${ }^{*}$ Os comportamento de Responsividade - Observar o contexto (OC), Diretividade - Engajar socialmente (ES) e de Intrusividade - Aproximar-se abruptamente (AAb) não foram codificados nesse contexto porque não eram aplicáveis à situação(estruturada)

\section{Discussão}

Observou-se que o estilo Diretivo foi o mais utilizado pelas avaliadoras em interação com as crianças, o que corrobora com a literatura sobre interação adulto-criança com TEA. Um estudo exploratório comparou a interação de pais e seus filhos (46 com TEA e 42 com desenvolvimento típico), utilizando um instrumento de intervenção na interação das díades (Parent Child Interaction Therapy - PCIT). Os resultados do estudo revelaram que, de maneira geral, os pais de crianças com TEA tendem a interagir com seus filhos de forma similar aos pais de crianças com desenvolvimento típico, porém os pais de crianças com TEA apresentam comportamento 
significativamente mais diretivo que os pais do grupo controle (Zlomke et al., 2019). Uma das possíveis explicações para isso é que crianças com TEA tendem a estabelecer interações pouco recíprocas e espontâneas e intencionais, o que faz com que o adulto tenda a adotar um estilo mais diretivo frente às limitadas respostas sociais infantis, na interação.

Um estudo longitudinal de Wagner et al. (2019) investigou a variação do comportamento social recíproco (VCR) de crianças com TEA. Participaram 602 indivíduos, divididos em quatro grupos, sendo estes: 1 . indivíduos com TEA; 2. irmãos de indivíduos com TEA; 3. indivíduos com ou sem TEA, mas com diagnóstico psiquiátrico; e 4. irmãos de indivíduos com diagnóstico psiquiátrico. Os participantes tinham idades entre 2,5 e 29 anos de idade. Os resultados do estudo revelaram que, embora a VCR tenha se mantido estável dentro dos grupos, observou-se uma diferença significativa entre os escores do grupo de indivíduos com TEA e os demais grupos investigados (VCR três desvios padrão abaixo de indivíduos sem TEA e sem diagnóstico psiquiátrico; dois desvios padrão abaixo de indivíduos (com ou sem TEA) e com diagnóstico psiquiátrico). Crianças com TEA tenderam a apresentar menos comportamentos sociais recíprocos quando comparadas a outras crianças.

No presente estudo, observou-se que, especificamente na brincadeira semiestruturada, a Diretividade atingiu frequências maiores do que no contexto de brincadeira livre. Neste último, o manual do instrumento orienta o avaliador a permitir que a criança explore o ambiente e os brinquedos à sua disposição de maneira mais autônoma; já no contexto de brincadeira semiestruturada, o avaliador é orientado a apresentar cada um dos objetos da caixa do avaliador individualmente, demonstrando à criança como operá-los, antes de entregar o brinquedo (sempre que possível) (Bosa \& Salles, 2018). Isso explica a alta frequência dos comportamentos de dirigir a atenção, demonstrar como se opera um objeto e tornar as brincadeiras atrativas nesse contexto de brincadeira.

Além disso, a maior ocorrência do comportamento de antecipar ações no contexto semiestruturado do que no contexto de brincadeira livre. Provavelmente isso ocorreu devido à natureza dos estímulos presentes nas brincadeiras semiestruturadas (predominantemente sensorial), o que pode acarretar uma sobrecarga sensorial (APA, 2013), quando apresentados subitamente. Embora todos os comportamentos do estilo intrusivo tenham ocorrido com baixa frequência, mesmo no contexto semiestruturado, o comportamento de não antecipar ações foi o mais frequente. Isso demonstra uma necessidade de melhor treinamento do avaliador, já que se trata de um contexto que deve ser mais dirigido por ele.

Já a baixa ocorrência dos comportamentos considerados intrusivos durante a interação das avaliadoras com os avaliados, sugere que o tempo de experiência destas com o instrumento PROTEA-R-NV talvez influencie positivamente na baixa ocorrência de condutas que dificultam a interação com essas crianças. O próprio manual do protocolo também orienta para a necessidade de o profissional evitar comportamentos intrusivos em função das possíveis respostas sensoriais atípicas dessas crianças (hipo ou hiperreatividade a estímulos simultâneos). Por exemplo, um estudo de Feldman et al. (2018) explorou a relação entre medidas de sensibilidade sensorial em crianças com e sem TEA (18 com TEA e 18 grupo controle) de oito a 17 anos. Os resultados revelaram que os níveis médios de respostas sensoriais no grupo com TEA foram significativamente maiores em hiporreação, hiperreação e busca sensorial, em relação aos controles com desenvolvimento típico. Ainda, ressalta- se que a baixa Intrusividade pode estar relacionada aos altos índices dos comportamentos de observar o contexto e compartilhar tópico, por parte das avaliadoras, considerando-se que esses comportamentos sugerem que as profissionais estavam atentas às demandas $\mathrm{e}$ interesses infantis.

Os comportamentos caracterizados como Responsividade no Manual de Codificação dos Estilos Interativos dos Avaliadores mais adotados pelas avaliadoras foram observar o contexto e compartilhar tópico. O comportamento de observar o contexto diz respeito ao avaliador considerar o que estava ocorrendo no ambiente antes da sua entrada. Refere-se também a pontuar que ele percebe as aparentes intenções da criança, mesmo que a estrutura da avaliação não permita seguir o interesse infantil naquele momento (e.g., precisar trocar o objeto, considerando o tempo de sessão máximo sugerido). A alta ocorrência desse comportamento pode ser explicada pela natureza do contexto livre de brincadeira. Nesse contexto, o avaliador é orientado a observar as explorações autônomas da criança antes de sugerir alguma brincadeira ou atividade (Bosa \& Salles, 2018), evitando, desse modo, a Intrusividade. Da mesma forma, a alta presença do comportamento de compartilhar tópico sugere que as avaliadoras priorizaram as demandas e aparentes interesses infantis na interação, permitindo que as crianças explorassem o ambiente livremente. Para Tomasello (1999/2003), por exemplo, as cenas de atenção compartilhada recorrentes no dia a dia das crianças favorecem a aquisição da linguagem no desenvolvimento infantil.

Por outro lado, ainda no contexto de brincadeira livre, os índices de ocorrência do comportamento de fazer perguntas em excesso (Intrusividade), sugerem que $\mathrm{o}$ avaliador tentou suprir com perguntas, as lacunas em que predomina o silêncio por parte da criança. Um estudo de metanálise de Ku et al., (2019) explorou as diferenças de comportamento parental de crianças com TEA e com desenvolvimento típico. Os resultados revelaram que pais de crianças com TEA apresentaram comportamentos significativamente mais "controladores" que os pais das outras crianças (Hedge's $g=0,53$; IC 95\%: $0,13-0,93 ; Z=2,60 ; p=0,009)$, os quais se manifestam 
por meio de comportamentos verbais (intrusão, ordens e comentários) não coerentes com o contexto.

Em síntese, o presente estudo identificou quais comportamentos devem ser mantidos no contexto de hora lúdica diagnóstica e quais podem ser evitados. A metodologia adotada para a investigação do perfil das avaliadoras participantes (observação sistemática de videogravações) permite uma análise mais acurada do comportamento, além da concordância entre juízes, que sugere a fidedignidade na operacionalização dos comportamentos descritos no Manual. Por outro lado, a análise de episódios interativos por unidade de tempo, ainda que tenha sido útil, pode ter prejudicado a observância de alguns comportamentos entre um episódio e outro.

A investigação dos comportamentos das crianças pode ser explorada em novos estudos a fim de permitir uma compreensão mais profunda sobre a influência recíproca dos comportamentos desses profissionais nos resultados da avaliação psicodiagnóstica. De qualquer modo, os resultados do estudo contribuem para a formação de profissionais nesse contexto, ao qualificar a conduta do avaliador de modo a facilitar a emergência, não apenas das dificuldades, mas também das potencialidades infantis na interação.

\section{Agradecimentos}

À Coordenação de Aperfeiçoamento Pessoal de Nível Superior (CAPES), pois foi através da bolsa de Mestrado que a presente pesquisa pode ser realizada com êxito; à Cleonice Alves Bosa, orientadora do projeto e pesquisa; e à Letícia Backes Schreiner, pelo apoio na coleta e análise dos dados.

\section{Financiamento}

Todas as fontes de financiamento para elaboração e produção do estudo (coleta, análise e interpretação dos dados, bem como, escrita dos resultados no presente no manuscrito) foram fornecidas pelo projeto de pesquisa com código do financiamento 001- Coordenação de Aperfeiçoamento Pessoal de Nível Superior (CAPES).

\section{Contribuições dos autores}

Todos os autores participaram da elaboração do manuscrito. Especificamente, a autora Gabriela Moreira Romeira participou da redação inicial do estudo - conceitualização, investigação, visualização, as autoras Gabriela Moreira Romeira e Letícia Backes Schreiner participaram da análise dos dados, e a autora Cleonice Alves Bosa participou da redação final do trabalho - revisão e edição.

\section{Disponibilidade dos dados e materiais}

Todos os dados e sintaxes gerados e analisados durante esta pesquisa serão tratados com total sigilo devido às exigências do Comitê de Ética em Pesquisa em Seres Humanos. Porém, o conjunto de dados e sintaxes que apoiam as conclusões deste artigo estão disponíveis mediante razoável solicitação ao autor principal do estudo.

\section{Conflito de interesses}

Os autores declaram que não há conflitos de interesses.

\section{Referências}

Akhtar, N., \& Tomasello, M. (1996). Two-year-olds learn words for absent objects and actions. British Journal of Developmental Psychology, 14(1), 79-93. https://doi.org/10.1111/j.2044-835X.1996.tb00695.x

American Psychiatric Association (2013). Diagnostic and Statistical Manual of Mental Disorders (5th ed.). American Psychiatric Publishing.

Aquino, F. S. B., \& Salomão, N. M. R. (2005). Estilos diretivos maternos apresentados a meninos e meninas. Estudos de Psicologia, 10(2), 223230. https://doi.org/10.1590/S1413- 294X2005000200009

Bakeman, R., \& Gottman, J. M. (1986). Observing interaction: An introduction to sequential analysis. Cambridge University Press.

Bosa, C. A. (1998). Affect, communication and self-stimulation in children with and without autism: A systematic observation study of joint attention and requesting behaviors. Unpublishedmaster'sthesis. University of London.

Bosa, C. A., Backes, B., Romeira, G. M., \& Zanon, R. B. (2017). Avaliação sociocomunicativa nos casos de suspeita de autismo: Diretrizes para a hora lúdica diagnóstica. Em C. A. Bosa, \& M. C. T. V. Teixeira, (Eds.). Autismo: Avaliação psicológica e neuropsicológica (pp. 83-104). Hogrefe.

Bosa, C. A., \& Salles, J. F. (2018). Sistema PROTEA-R de avaliação da suspeita de Transtorno do Espectro Autista. Vetor.

Bosa, C. A., \& Souza, A. D. (2007). Interação mãe-criança e desenvolvimento atípico: A contribuição da observação sistemática. Em C. A. Piccinini, \& M. L. S. Moura, (Eds.). Observando a interação pais-bebê-criança (pp. 235-257). Casa do Psicólogo.

Bosa, C. A., \& Zanon, R. (2016). Psicodiagnóstico e transtorno do espectro autista. Em D. Hutz, C. Bandeira, Trentini \& J. Krug (Eds.), Psicodiagnóstico (pp. 308-322). Artmed.

Bosa, C. A., Zanon, R., \& Backes, B. (2016). Autismo: construção de um protocolo de avaliação do comportamento da criança - PROTEA-R. Psicologia: Teoria e Prática, 18(1), 194-205. https://doi.org/10.15348/1980-6906/psicologia.v18n1p194-205

Czermainski, F. R., Riesgo, R. S., Guimarães, L. S. P., Salles, J. F., \& Bosa, C. A. (2014). Déficits de funções executivas em crianças e adolescentes com Transtorno do Espectro do Autismo. Paidéia - Cadernos de Psicologia e Educação, 24(57), 85-94. https://doi. org/10.1590/1982-43272457201411

Delaherche, E., Chetouani, M., Mahdhaoui, A., Saint-Georges, C., Viaux, S., \& Cohen, (2012). Interpersonal synchrony: A survey of evaluation methods across disciplines. IEEE Transactionson Affective Computing, 3(3) 1-20. https://doi.org/10.1109/T- AFFC.2012.12 
Dos Santos, A. C., Ribeiro, I. F., Garotti, M. F., \& Bosa, C. A. (2015). Intervention in autism: Social engagement implemented by caregivers. Paidéia - Cadernos de Psicologia e Educação, 25(60), 67-75. https://doi.org/10.1590/1982-43272560201509

Fagundes, A. J. D. F. M. (2015). Descrição, definição e registro de comportamento (17th ed.). Edicon.

Feldman, J., Kuang, W., Conrad J. G., Tu, A., Santapuram, P., Simon, D. M., et al. (2018). Briefreport: Differences in multisensory integration covary with sensory responsiveness in children with and without Autism Spectrum Disorder. Journal of Autism and Developmental Disorders, 49(1), 397-403. https://doi.org/10.1007/s10803-018-3667-x

IBM Corp. Released. (2016). IBM SPSS Statistics for Windows (Version 24.0) [Computer software]. IBM Corp.

Krug, J. S., \& Bandeira, D. R. (2016). Critérios de análise do brincar infantil na entrevista lúdica diagnóstica. Em C. S. Hutz, D. R. Bandeira, C. M. Trentini \& J. S. Krug (Eds.), Psicodiagnóstico (pp. 16-20). Artmed.

Ku, B., Stinson, J. D., \& MacDonald, M. (2019). Parental behavior comparisons between parentes of children with Autism Spectrum Disorder and parentes of children without Autism Spectrum Disorder: A meta-analysis. Journal of Child and Family Studies, 28(6), 14451460. https://doi.org/10.1007/s10826-019-01412-w

Leclère, C., Viaux, S., Avril, M., Achard, C., Chetouani, M., Missonnier, S., \& Cohen, D. (2014). Why synchrony matters during motherchild interactions: A systematic review. PLoS ONE, 9(12), 1-34. https://doi.org/10.1371/journal.pone.0113571

Marques, D. F., \& Bosa, C. A. (2015). Protocolo de avaliação de crianças com autismo: Evidências de validade e de critério. Psicologia: Teoria e Pesquisa, 31(1), 43-51. https://doi.org/10.1590/0102-37722015011085043051

Meimes, M. A. (2014). Interação mãe-criança e autismo: A contribuição dos fatores psicossociais. [Unpublished master's thesis]. Universidade Federal do Rio Grande do Sul.

Mugzach, O., Peleg, M., Bagley, S. C., Guter, S. J., Cook, E. H., \& Russ, B. (2015). An ontology for Autism Spectrum Disorder (ASD) to infer ASD phenotypes from Autism Diagnostic Interview-Revised data. Journal of Biomedical Informatics, 56, 333-347. https://doi. org/10.1016/j.jbi.2015.06.026

Paavola-Ruotsalainen, L., Lehtosaari, J., Palomäki, J., \& Tervo, I. (2017). Maternal verbal responsiveness and directiveness: consistency, stability, and relations to child early linguistic development. Journal of Child Language, 45(2), 319-339. https://doi.org/10.1017/ S030500091700023X.

Seabra, A. G., Bosa, C. A., \& Dias, N. M. (2017). Funções executivas: Definições do construto, principais alterações no Transtorno do Espectro Autista e instrumentos de avaliação no contexto nacional. Em C. A. Bosa \& M. C. T. V. Teixeira (Eds.), Autismo: Avaliação psicológica e neuropsicológica (pp. 179-203). Hogrefe.

Teixeira, M. C. T. V., Carreiro, L. R. R., Cantiere, C. N., \& Baraldi, G. S. (2017). Perfil cognitivo e comportamental do Transtorno do Espectro Autista. Em C. A. Bosa \& M. C. T. V. Teixeira (Eds.), Autismo: Avaliação psicológica e neuropsicológica (pp. 29-41). São Paulo.

Tomasello, M. (1999/2003). Origens culturais da aquisição do conhecimento humano. (C, Berliner, Trans.). Martins Fontes (Original work published in 1999).

Volkmar, F., Chawarska, K., \& Klin, A. (2004). Autism in infancy and early childhood. Annual Review of Psychology, 56, 315-336. https://doi. org/10.1146/annurev.psych.56.091103.070159

Wagner, R. E., Zhang, Y., Gray, T., Abbacchi, A., Cormier, D., Todorov, A., Constantino, J. N (2019). Autism-related variation in reciprocal social behavior: A longitudinal study. Child Development, 90(2), 441- 451. https://doi.org/10.1111/cdev.13170

Zanon, R. B., Backes, B., \& Bosa, C. A. (2014). Identificação dos primeiros sintomas do autismo pelos pais. Psicologia: Teoria e Pesquisa, 30(1), 25-33. https://doi.org/10.1590/S0102- 37722014000100004

Zlomke, K., Bauman, S., \& Edwards, G. (2019). An exploratory study of the utility of the dyadic parent-child interaction coding system for children with Autism Spectrum Disorder. Journal of Developmental and Physical Disabilities, 31(4), 501-518. https://doi.org/10.1007/ s10882-018-9648-3

Zyga, O., Russ, S., \& Dimitropoulos, A. (2015). Assessment of pretend play in Prader - Willi syndrome: A direct comparison to Autism Spectrum Disorder. Journal of Autism and Developmental Disorders, 45(4), 975-987. https://doi.org/10.1007/s10803-014-2252-1

\section{Sobre as autoras}

Gabriela Moreira Romeira, psicóloga e Mestre em Psicologia pela Universidade Federal do Rio Grande do Sul (UFRGS).

Letícia Backes Schreiner, graduanda em Psicologia na Universidade Federal do Rio Grande do Sul (UFRGS) e bolsista de iniciação científica no Núcleo de Estudos e Pesquisas em Transtorno do Desenvolvimento (NIEPED).

Cleonice Alves Bosa, psicóloga e Profa. Titular do Programa de Pós-graduação em Psicologia do Desenvolvimento e da Personalidade (UFRGS).

\section{Como citar este artigo}

Romeira G. M., Schreiner, L. B., \& Bosa, C. A. (2021). Avaliação Psicológica de Crianças com Suspeita de TEA: Perfil Interativo dos Avaliadores. Avaliação Psicológica, 20(1), 43-51. http://dx.doi.org/10.15689/ap.2021.2001.19578.05 